\title{
LIMIT PROPERTIES OF POISSON KERNELS OF SIEGEL DOMAINS OF TYPE II
}

\author{
BY
}

\section{LAWRENCE J. DICKSON}

\begin{abstract}
The results of $[1]$ concerning tight $C_{0}^{*}$ limit of the Poisson kernel of a tube domain, as its parameter converges to a point on the cone boundary, are extended under certain hypotheses to Siegel domains of type II. In the case where the domain is polytopic, almost everywhere convergence of the $L^{p}$ Poisson integral to its boundary values is obtained. Examples and further conjectures conclude the paper.
\end{abstract}

I. Notation and basics. In this paper we extend some of the results of [1] to Siegel domains of type II (which we will henceforth call "Siegel domains"). This paper is a sequel to [1], from which we draw our basic notation. Also we will refer freely to parallel results and proofs in that paper.

We will make use of two notational conveniences. The lower-case letter $c$, with or without subscripts, will always denote a positive constant or function dependent only on the dimensions $m$ and $n$ and the original Siegel domain $\Omega=$ $\Omega_{\Gamma, \Phi}$ under consideration (see below). Secondly, we will omit explicit references to degenerate cases (such as $n=0$ ) while including them in our theorems: the interpretation of the $0 \times k$ matrices, $0 \times 0$ determinants, etc., arising in these cases is obvious.

1.1. Definitions. Let $\mathbf{C}^{m}=\mathbf{R}^{m} \oplus i \mathbf{R}^{m}$ and $\mathbf{C}^{n}$ be finite-dimensional vector spaces over $\mathbf{C}$, with (fixed) inner products induced by the direct sum, and $\mathbf{R}^{m}$ a (fixed) real form of $\mathbf{C}^{m}$. A Siegel domain of class $(m, n)$ is a domain $\Omega$ of $\mathbf{C}^{m+n}=\mathbf{R}^{m} \oplus i \mathbf{R}^{m} \oplus \mathbf{C}^{n}$ defined as follows [2], [4] :

$$
\Omega=\Omega_{\Gamma, \Phi}=\left\{(x+i y+i \Phi(z), z): x \in \mathbf{R}^{m}, y \in \Gamma, z \in \mathbf{C}^{n}\right\}
$$

where $\Phi: \mathbf{C}^{n} \times \mathbf{C}^{n} \rightarrow \mathbf{C}^{m}$ is a Hermitian form (relative to $\mathbf{R}^{m}$ ) satisfying, for the proper cone $\Gamma$,

Received by the editors October 22, 1974.

AMS (MOS) subject classifications (1970). Primary 31 B10, 52A20; Secondary 31 B25, 32 F05.

Key words and phrases. Szego and Poisson kernels, cones, Hermitian forms, nilpotent groups, limit cones, tight $C_{0}^{*}$ limit, weak-type operator. 


$$
z \neq 0 \Rightarrow \Phi(z) \in \bar{\Gamma}-\{0\} .
$$

Here we write $\Phi(z)$ for $\Phi(z, z)$.

The distinguished boundary of $\Omega$ is $\Omega_{0}=\left\{(x+i \Phi(z), z): x \in \mathbf{R}^{m}, z \in \mathbf{C}^{n}\right\}$.

The nilpotent group $N$ of $\Omega$ is the group which is set-theoretically identical with $\mathbf{R}^{m} \oplus \mathbf{C}^{n}$ but which possesses the group operation

$$
(x, z) \circ(u, w)=(x+u-2 \operatorname{Im} \Phi(z, w), z+w) .
$$

Its operation on $\Omega$ is defined by (3) and

$$
(x, z)[(i y, 0)]=(x+i y+i \Phi(z), z), \quad y \in \bar{\Gamma} .
$$

We set up the canonical identification of $\mathrm{R}^{m} \oplus \mathrm{C}^{n}, N$, and $\Omega_{0}$ by

(5) $(x, z) \in \mathbf{R}^{m} \oplus \mathbf{C}^{n} \leftrightarrow(x, z) \in N \leftrightarrow(x, z)[(0,0)]=(x+i \Phi(z), z) \in \Omega_{0}$.

The canonical measures $d \mu$ on $\Omega_{0}$ and $d \nu$ on $N$ are those induced by canonical Lebesgue measure $d \lambda^{m+2 n}$ on $\mathbf{R}^{m} \oplus \mathbf{C}^{n}$ under this identification; $d v$ is Haar measure on $N$.

The group $N$ is transitive on $\Omega_{0}$ and acts on $\Omega$ (or $\bar{\Omega}$ ). $\mathbf{R}^{m} \oplus \mathbf{C}^{n}$ may be identified with the underlying vector space of the Lie algebra of $N$, and then the canonical identification becomes the exponential map. All the structure in the above definition is invariant under transformations of the form $Q \oplus U$, where $Q$ is real orthogonal on $\mathrm{R}^{m} \oplus i \mathrm{R}^{m}$ and $U$ is unitary on $\mathbf{C}^{n}$ : we shall therefore freely identify Siegel domains which are related by such transformations.

1.2. Definition (SEE [2]). The Szegö kernel $S(Z, W)=S_{W}(Z)$ of $\Omega$ is the reproducing kernel of $H^{2}(\Omega)$, where the norm on $H^{2}(\Omega)$ is defined as the $L^{2}$ norm of the $\Omega_{0}$ boundary values using the measure $d \mu$. The Poisson kernel $P(Z, W)=P_{W}(Z), Z \in \Omega_{0}, W \in \Omega$ is defined by

$$
P_{W}(Z)=\left\|S_{W}\right\|_{2}^{-2}\left|S_{W}(Z)\right|^{2} .
$$

Using Haar measure and the uniqueness of $S$, one easily gets $S(\nu(Z), \nu(W))$ $=S(Z, W)$ for $\nu \in N$, and hence $P(\nu(Z), \nu(W))=P(Z, W)$. Since for our purposes we may assume $Z \in \Omega_{0}$, we need only investigate the functions $S$ and $P$, where

$$
\begin{aligned}
& S(v ; x, z)=S(x+i \Phi(z), ; z ; i v, 0), \\
& P(v ; x, z)=P(x+i \Phi(z), z ; i v, 0), \quad v \in \Gamma, x \in \mathbf{R}^{m}, z \in \mathbf{C}^{n} .
\end{aligned}
$$

The usual method yields [2]

$$
P(v ; x, z)=|S(v ; x, z)|^{2} / S(2 v ; 0,0) .
$$

1.3. Claim. Suppose $A \in \mathrm{GL}(m, \mathrm{R})$ and $B \in \mathrm{GL}(n, \mathrm{C})$, and $\Omega=\Omega_{\Gamma, \Phi}$ 
is a Siegel domain of class $(m, n)$. Then $\Omega^{\prime}=(A \oplus B) \Omega$ is also a Siegel domain: in fact $\Omega^{\prime}=\Omega_{\Gamma^{\prime}, \Phi^{\prime}}$, where $\Gamma^{\prime}=A \Gamma$ and $\Phi^{\prime}=A \Phi^{B}$. Here $A \Phi^{B}$ is defined by

$$
\left(A \Phi^{B}\right)(z, w)=A \Phi\left(B^{-1} z, B^{-1} w\right), \quad z, w \in \mathrm{C}^{n}
$$

Also

$$
\begin{aligned}
& S_{\Omega^{\prime}}(A v ; A x, B z)=(\operatorname{det} A)^{-1}|\operatorname{det} B|^{-2} S_{\Omega}(v ; x, z), \\
& P_{\Omega^{\prime}}(A v ; A x, B z)=(\operatorname{det} A)^{-1}|\operatorname{det} B|^{-2} P_{\Omega}(v ; x, z) .
\end{aligned}
$$

Proof. Elementary, using uniqueness of $S$.

1.4. Claim (explicit formulas). Define $\mathrm{C}_{n}$ to be the $n \times n$ complex matrices, $H_{n} \subset \mathrm{C}_{n}$ the Hermitian matrices, $H_{n}^{+} \subset H_{n}$ the positive definite matrices. For $\alpha \in \Gamma^{*}$ define $\Phi(\alpha) \in H_{n}^{+}$by requiring $\langle\Phi(z, w), \alpha\rangle=z^{t} \Phi(\alpha) \bar{w}$. Then

$$
S(v ; x, z)=2^{n-m} \pi^{-m-n} \int_{\Gamma^{*}} e^{-\langle\Phi(z)+v-i x, \alpha\rangle} \operatorname{det} \Phi(\alpha) d \lambda^{m}(\alpha) .
$$

Let $\hat{S}(v ; \alpha, \hat{z})$ denote the $m+2 n$-dimensional Fourier transform of $S$ with respect to $x$ and $z$, where $z$ is treated as a $2 n$-dimensional real variable with real inner product $\langle z, w\rangle^{\mathrm{R}}=\operatorname{Re}\langle z, w\rangle$, and similarly for $P(v ; \alpha, z)$. Then

$$
\hat{S}(v ;-\alpha, \hat{z})=2^{n} \chi_{\Gamma^{*}}(\alpha) \exp \left[-2 \pi\left(\langle v, \alpha)+1 / 4 \Phi(\alpha)^{-1}(\hat{z})\right)\right],
$$

where $\Phi(\alpha)^{-1}(w)=w^{t} \Phi(\alpha)^{-1} \bar{w}$; and

$\hat{P}(v ; \alpha, \hat{z})=c(v) \int_{\Gamma_{\alpha}^{*}} e^{-4 \pi(v, \beta)}$

$$
\begin{aligned}
\cdot \int_{C^{n}} \exp \left\{-\frac{\pi}{2}[\Phi(\beta+\right. & \left.\frac{\alpha}{2}\right)^{-1}\left(w+\frac{\hat{z}}{2}\right) \\
& \left.\left.+\Phi\left(\beta-\frac{\alpha}{2}\right)^{-1} \mid\left(w-\frac{\hat{z}}{2}\right)\right]\right\} d \lambda^{2 n}(w) d \lambda^{m}(\beta)
\end{aligned}
$$

where $\Gamma_{\alpha}^{*}=\left(\Gamma^{*}+\alpha / 2\right) \cap\left(\Gamma^{*}-\alpha / 2\right) \subset \Gamma^{*}$, and $c(v)$ takes such a value that $\hat{P}(v ; 0,0)=1$.

PROOF. Formula (12) is essentially the same as Gindikin's formula given in [2]: we have merely integrated the expression for $L(\alpha)$ in that paper. The Fourier inversion formula applied to (12) immediately gives us the Fourier transform with respect to $x$ alone:

$$
\hat{S}(v ;-\alpha, z)=4^{n} \chi_{\Gamma^{*}}(\alpha) e^{-2 \pi(\Phi(z)+v, \alpha\rangle} \operatorname{det} \Phi(\alpha) .
$$

Multiplying by $\exp (2 \pi i \operatorname{Re}\langle\hat{z}, z\rangle)$ and integrating over $z \in \mathrm{C}^{n}$ yields (13) upon diagonalizing $\Phi(\alpha)$ by a unitary transformation of $C^{n}$. (14) follows from (8) and 
the product formula for Fourier transforms, using the fact that the Poisson kernel has unit integral.

1.5. Claim. Let $f$ be a function of $L^{p}(N) \cong L^{p}\left(\mathbf{R}^{m} \oplus \mathbf{C}^{n}\right) \cong L^{p}\left(\Omega_{0}\right)$, $1 \leqslant p \leqslant \infty$. For $W \in \Omega$ define $P f(W)=\left\langle f, P_{W}\right\rangle$ to be the Poisson integral of $f$; if $f \in L^{2}$, define $S f(W)=\left\langle f, S_{W}\right\rangle$ to be the Szegö-integral or holomorphic part of $f$.

Then for $(x, z) \in N, v \in \Gamma$, and convolution in $N$, we have

$$
\begin{aligned}
& S f((x, z)[(i v, 0)])=\left(f * S_{v}\right)(x, z) ; \\
& P f((x, z)[(i v, 0)])=\left(f * P_{v}\right)(x, z) .
\end{aligned}
$$

Proof. (15) follows from (7), the group invariance of $S$, and the fact that $\bar{S}(v ; x, z)=S(v ;-x,-z)$. The proof of $(16)$ is similar.

1.6. Definition. Let $m, n$ be nonnegative integers, $C$ the set of all proper cones of $\mathbf{R}^{m}$ with the cone metric $[1,2.2], \Theta$ the set of all Hermitian forms $\Phi$ : $\mathbf{C}^{n} \times \mathbf{C}^{n} \rightarrow \mathbf{R}^{m} \oplus i \mathbf{R}^{m}$ with a linear norm. Define

$$
G=\left\{(\Gamma, \Phi ; v) \in C \times \Theta \times \mathbf{R}^{m}: \Phi \text { is } \Gamma \text {-definite, } v \in \Gamma\right\},
$$

with topology induced by the product topology for $C \times \Theta \times \mathbf{R}^{m}$.

Suppose $\gamma \in G, \gamma=(\Gamma, \Phi ; v)$; let $T \subset \mathbf{R}^{m}$ and $U \subset \mathbf{C}^{n}$ be subspaces of real dimension $k$ and complex dimension $l$ respectively. If $\Omega=\Omega_{\Gamma, \Phi}$, we define

$$
\begin{gathered}
S(\gamma ; x, z)=S_{\gamma}(x, z)=S_{\Omega}(v ; x, z) \\
P(\gamma ; x, z)=P_{\gamma}(x, z)=P_{\Omega}(v ; x, z) \\
P^{T, U}\left(\gamma ; x_{1}, z_{1}\right)=P_{\gamma}^{T, U}\left(x_{1}, z_{1}\right) \\
=\int_{T^{\perp}} \int_{U^{\perp}} P\left(\gamma ; x_{1}+x_{2}, z_{1}+z_{2}\right) d \lambda^{2 n-2 l}\left(z_{2}\right) d \lambda^{m-k}\left(x_{2}\right),
\end{gathered}
$$

for $x \in \mathbf{R}^{m}, x_{1} \in T, z \in \mathbf{C}^{n}, z_{1} \in U$.

1.7. ThEOREM (KERNEL CONTINUITY). Let $T, U$ be fixed, notation as in 1.6 .

Then the map from $G$ into $C_{0}\left(\mathbf{R}^{m} \oplus \mathbf{C}^{n}\right)$ defined by $\boldsymbol{\gamma} \mapsto S_{\boldsymbol{\gamma}}$ maps $G$ continuously into $L^{p}\left(\mathbf{R}^{m} \oplus \mathbf{C}^{n}\right), 2 \leqslant p \leqslant \infty$. The map from $G$ into $C_{0}(T \oplus U)$ defined by $\gamma \mapsto P_{\gamma}^{T, U}$ sends $G$ continuously into $L^{p}(T \oplus U), 1 \leqslant p \leqslant \infty$.

Proof. The kernels are clearly $C_{0}$, so it will be enough to show the first claim for $L^{2}$ and $L^{\infty}$, and the second for $L^{1}$ and $L^{\infty}$. To do this, we go to the Fourier transform side. All the transformed kernels are exponentially decreasing, and uniformly so over compact subsets of $G$. Since $\gamma \mapsto \hat{S}_{\gamma}$ is clearly $L^{1}$ and $L^{2}$ continuous on compact sets, it is continuous in these norms, and the first 
claim follows. The $L^{1}$ cases of the second claim follow from (8), Fubini's theorem, and the $L^{2}$ case of the first. The $L^{\infty}$ case follows from the $L^{1}$ behaviour of the Fourier transform of $P_{\gamma}^{T, U}$, which is just the restriction of $\hat{P}_{\gamma}$ to $T \oplus U$.

II. Tight $C_{0}^{*}$ convergence of the Poisson kernel as its parameter approaches a boundary point. In this section $p$ will denote a point in $\bar{\Gamma}$. By $p^{\perp}$ we will mean the subspace $[\{p\}]^{\perp}$; and $\Gamma^{*}(p)=\operatorname{Inn}\left(p^{\perp} \cap \bar{\Gamma}^{*}\right), T_{3}(p)=\left[\Gamma^{*}(p)\right]$, $T_{1}(p)=\left[\Gamma_{p}\right], T_{2}(p)=\left[T_{1}(p) \oplus T_{3}(p)\right]^{\perp}$. Here brackets around a subset of a vector space denote the linear span of that subset, and $\bar{\Gamma}_{p}$ is the largest closed subcone of $\bar{\Gamma}$ such that $p \in \operatorname{Inn} \bar{\Gamma}_{p}=\Gamma_{p}$. Thus, at $p$, the cone $\bar{\Gamma}$ is "flat" in $T_{1}$ directions, "sharp" in $T_{2}$ directions, and "rounded" in $T_{3}$ directions. Other notation is as in [1].

2.1. Definition. Let $\Omega=\Omega_{\Gamma, \Phi}$ be a Siegel domain of class $(m, n)$, and let $p \in \bar{\Gamma} . p$ is called a nicely differentiable ("nice") point of $\bar{\Gamma}$ with respect to $\Phi$ if the following hold:

(a) $p$ is a "nice" point of $\bar{\Gamma}[1,2.6]$;

(b) there exists $\alpha \in \bar{\Gamma}^{*}(p)$ such that $\Phi(\operatorname{Ker} \Phi(\alpha)) \subset T_{1}(p)$.

Here the systematic confusion about the domain of $\Phi$ arises: we consider $\Phi(\alpha)$ as a transformation of $C^{n}$, and the outer $\Phi$ as in (2). Note that if, as "usually" happens, $\bar{\Gamma}_{p}=\{q \in \bar{\Gamma}:\langle q, \alpha\rangle=0\}$ some $\alpha \in \bar{\Gamma}^{*}(p)$, then (b) is automatically satisfied. Also note that (since $\operatorname{Ker} \Phi\left(\alpha_{0}+t \beta\right)=\left(\operatorname{Ker} \Phi\left(\alpha_{0}\right)\right) \cap$ $(\operatorname{Ker} \Phi(\beta))$ for $\left.\alpha_{0, \beta} \in \bar{\Gamma}^{*}, t>0\right) \operatorname{Ker} \Phi(\alpha)=\bigcap_{\beta \in \bar{\Gamma}^{*}(p)} \operatorname{Ker} \Phi(\beta)$ for every $\alpha \in \Gamma^{*}(p)$.

2.2. Claim. Let notation be as above, $p$ a "nice" point, and let $T_{j}=$ $T_{j}(p)$. Define $U_{1}=\operatorname{Ker} \Phi(\beta) \subset \mathrm{C}^{n}$ for $\beta \in \Gamma^{*}(p)$ and let $U_{2}=U_{1}^{\perp}$. If $\alpha=$ $\alpha_{1}+\alpha_{2}+\alpha_{3} \in \bar{\Gamma}^{*}, \alpha_{j} \in T_{j}$, we have

(21) $\Phi(\alpha)=\left(\begin{array}{cc}A\left(\alpha_{1}\right) & B_{1}\left(\alpha_{1}\right) \\ B_{1}^{*}\left(\alpha_{1}\right) & C_{1}\left(\alpha_{1}\right)\end{array}\right)+\left(\begin{array}{cc}0 & B\left(\alpha_{2}\right) \\ B^{*}\left(\alpha_{2}\right) & C_{2}\left(\alpha_{2}\right)\end{array}\right)+\left(\begin{array}{cc}0 & 0 \\ 0 & C\left(\alpha_{3}\right)\end{array}\right) \geqslant 0$,

where the $A$ 's, $B$ 's and $C$ 's are linear functions of the $\alpha_{j}$ 's.

Now let $\tau_{j}$ be the orthogonal projection of $\mathrm{R}^{m}$ onto $T_{j}$ and $\pi_{j}$ the orthogonal projection of $\mathbf{C}^{n}$ onto $U_{j}$. For $\delta>0$ define the standard differentiator $(A \oplus B)_{\delta}=A_{\delta} \oplus B_{\delta}$, where $A_{\delta}=\tau_{1}+\delta^{-1} \tau_{2}+\delta^{-2} \tau_{3}$ and $B_{\delta}=\pi_{1}+\delta^{-1} \pi_{2}$. Then if $p$ is a "nice" point with respect to $\Phi$ and $\Gamma_{0}=\lim _{\delta \rightarrow 0} A_{\delta} \Gamma$, then $\Phi_{0}=$ $\lim _{\delta \rightarrow 0} A_{\delta} \Phi^{B} \delta$ exists, is $\Gamma_{0}$-definite, and is defined by

$$
\Phi_{0}(\alpha)=\left(\begin{array}{ll}
A\left(\alpha_{1}\right) & B\left(\alpha_{2}\right) \\
B^{*}\left(\alpha_{2}\right) & C\left(\alpha_{3}\right)
\end{array}\right) \geqslant 0 \quad \text { if } \alpha=\alpha_{1}+\alpha_{2}+\alpha_{3} \in \bar{\Gamma}_{0}^{*}
$$


Furthermore, $C\left(\alpha_{3}\right)>0$ for $\alpha_{3} \in \Gamma^{*}(p)$ and $A\left(\alpha_{1}\right)>0$ for $\alpha_{1} \in \operatorname{Inn} \tau_{1}\left(\bar{\Gamma}^{*}\right)=$ $\tau_{1}\left(\Gamma^{*}\right)$.

Proof. Since $\alpha_{2}, \alpha_{3} \perp T_{1}$ the zeros in the top left in (21) follow from 2.1(b). Since $\Gamma^{*}(p)$ spans $T_{3}$, it follows that $U_{1} \subset \operatorname{Ker} \Phi\left(\alpha_{3}\right) \forall \alpha_{3} \in T_{3}$, and the form of $\Phi\left(\alpha_{3}\right)$ is justified. $\Phi(\alpha) \geqslant 0$ follows since $\Phi$ is $\Gamma$-definite. The existence and expression for $\Phi_{0}$ follow by plugging in the definitions, and its $\Gamma_{0}$-definiteness follows. Since $U_{1}=\operatorname{Ker} \Phi(\alpha) \forall \alpha \in \Gamma^{*}(p), C\left(\alpha_{3}\right)>0$ must hold if $\alpha_{3} \in \Gamma^{*}(p)$. If $\alpha_{1}+\alpha_{2}+\alpha_{3}=\alpha \in \Gamma^{*}, 0 \neq z_{1} \in U_{1}$, then $0 \neq$ $\Phi(\alpha)\left(z_{1}\right)=z_{1}^{t} A\left(\alpha_{1}\right) \bar{z}_{1}$, so $A\left(\alpha_{1}\right)>0$. This completes the proof.

2.3. Definition. Let notation be as above. $p \in \bar{\Gamma}$ is regular with respect to $\Phi$ if the following hold:

(a) $p$ is a "nice" point of $\bar{\Gamma}$ with respect to $\Phi$,

(b) $p$ is a regular point of $\bar{\Gamma}[1,2.10]$,

(c) if $\Gamma_{0}^{*} \cap\left(T_{1}+\alpha_{2}+\alpha_{3}\right) \neq \varnothing$ then there exists $\alpha_{1} \in T_{1}$ such that $\alpha_{1}$ $+\alpha_{2}+\alpha_{3} \in \bar{\Gamma}_{0}^{*}$ and $A\left(\alpha_{1}\right)=B\left(\alpha_{2}\right) C\left(\alpha_{3}\right)^{-1} B^{*}\left(\alpha_{2}\right)$.

Note that the right-hand expression in (c) makes sense, since $\tau_{3} \Gamma_{0}^{*}=$ $\Gamma^{*}(p)$. Also if $\alpha_{1}$ satisfies (c) and $\alpha_{1}^{\prime} \in T_{1}$ we have $\alpha_{1}^{\prime}+\alpha_{2}+\alpha_{3} \in \bar{\Gamma}_{0}^{*}$ only if (23)

$$
\begin{aligned}
\left(\begin{array}{cc}
I & -B\left(\alpha_{2}\right) C\left(\alpha_{3}\right)^{-1} \\
0 & I
\end{array}\right)\left(\begin{array}{cc}
A\left(\alpha_{1}^{\prime}\right) & B\left(\alpha_{2}\right) \\
B^{*}\left(\alpha_{2}\right) & C\left(\alpha_{3}\right)
\end{array}\right)\left(\begin{array}{cc}
I & 0 \\
-C\left(\alpha_{3}\right)^{-1} B^{*}\left(\alpha_{2}\right) & I
\end{array}\right) \\
=\left(\begin{array}{cc}
A\left(\alpha_{1}^{\prime}\right)-B\left(\alpha_{2}\right) C\left(\alpha_{3}\right)^{-1} B^{*}\left(\alpha_{2}\right) & 0 \\
0 & C\left(\alpha_{3}\right)
\end{array}\right) \geqslant 0
\end{aligned}
$$

hence since $C\left(\alpha_{3}\right) \geqslant 0$ we get

$$
\alpha_{1}^{\prime}+\alpha_{2}+\alpha_{3} \in \bar{\Gamma}_{0}^{*} \Rightarrow A\left(\alpha_{1}^{\prime}-\alpha_{1}\right) \geqslant 0 .
$$

But if $\alpha_{1}^{\prime}$ is the base point of the cone $\left(\Gamma_{0}^{*}-\alpha_{2}-\alpha_{3}\right) \cap T_{1}$, then $\alpha_{1}-\alpha_{1}^{\prime} \in$ $\bar{\Gamma}_{p}^{*}$ by $[1,2.11]$; hence $A\left(\alpha_{1}-\alpha_{1}^{\prime}\right) \geqslant 0$ and $A\left(\alpha_{1}\right)=A\left(\alpha_{1}^{\prime}\right)$. Therefore we may assume that the $\alpha_{1}$ of $(\mathrm{c})$ is the base point of $\left(\bar{\Gamma}_{0}^{*}-\alpha_{2}-\alpha_{3}\right) \cap T_{1}$.

2.4. Lemma. Suppose $p \in \bar{\Gamma}$ is regular with respect to $\Phi$, with notation as above. Define the class $\left(m_{1}, n_{1}\right)$ Siegel domain

$$
\Omega_{p}=\Omega_{\Gamma_{p}, \Phi_{p}} \subset T_{1} \oplus i T_{1} \oplus U_{1} ; \quad \Phi_{p}\left(\alpha_{1}\right)=A\left(\alpha_{1}\right) \text { for } \alpha_{1} \in T_{1} .
$$

Then if $\gamma_{0}=\left(\Gamma_{0}, \Phi_{0} ; v\right)$ and $\tau_{1}(v)=p$, we have

$$
P^{T_{1}, U_{1}}\left(\gamma_{0} ; x_{1}, z_{1}\right)=P_{\Omega_{p}}\left(p ; x_{1}, z_{1}\right), \quad \forall\left(x_{1}, z_{1}\right) \in T_{1} \oplus U_{1} .
$$

Proof. Since both functions are continuous with integrable Fourier trans- 
forms, we need only prove these are equal. Let $\left(\beta_{2}, \beta_{3}\right) \in \Delta=\left(\tau_{2}+\tau_{3}\right)\left(\Gamma^{*}\right)$. If $\widetilde{\beta}_{1}=\widetilde{\beta}_{1}\left(\beta_{2}, \beta_{3}\right)$ is the base point of $\left(\bar{\Gamma}^{*}-\beta_{2}-\beta_{3}\right) \cap T_{1}$, we have by 2.3 that $A\left(\widetilde{\beta}_{1}\right)=B\left(\beta_{2}\right) C\left(\beta_{3}\right)^{-1} B^{*}\left(\beta_{2}\right)$. Now (see (13)) define the quadratic form

$$
L_{\Phi_{0}}(\beta, \alpha ; w, \hat{z})=\Phi_{0}\left(\beta+\frac{\alpha}{2}\right)^{-1}\left(w+\frac{\hat{z}}{2}\right)+\Phi_{0}\left(\beta-\frac{\alpha}{2}\right)^{-1}\left(w-\frac{\hat{z}}{2}\right) .
$$

If we require $\alpha_{j}, \beta_{j} \in T_{j} ; w_{j}, \hat{z}_{j} \in U_{j} ; \beta=\beta_{1}+\beta_{2}+\beta_{3}, w=w_{1}+w_{2}$, and $\eta=\beta_{1}-\tilde{\beta}_{1}\left(\beta_{2}, \beta_{3}\right)$, then

$$
\begin{aligned}
L_{\Phi_{0}}\left(\beta, \alpha_{1} ; w, \hat{z}_{1}\right)= & L_{\Phi_{p}}\left(\eta, \alpha_{1} ; w_{1}-\bar{B}\left(\beta_{2}\right) \bar{C}\left(\beta_{3}\right)^{-1} w_{2}, \hat{z}_{1}\right) \\
& +2 w_{2}^{t} C\left(\beta_{3}\right)^{-1} \bar{w}_{2},
\end{aligned}
$$

where the lower-dimensional $L_{\Phi_{p}}$ is defined as in (27).

Hence the inner integral of (14) is evaluated

$$
\begin{aligned}
& \int_{C^{n}} \exp \left(-\frac{\pi}{2} L_{\Phi_{0}}\left(\beta, \alpha_{1} ; w, \hat{z}_{1}\right)\right) d \lambda^{2 n}(w) \\
&=\int_{U_{2}} \exp \left(-\pi w_{2}^{t} C\left(\beta_{3}\right)^{-1} \bar{w}_{2}\right) \\
& \quad \cdot \int_{U_{1}} \exp \left(-\frac{\pi}{2} L_{\Phi_{p}}\left(\eta, \alpha_{1} ; w_{1}-\bar{B}\left(\beta_{2}\right) \bar{C}\left(\beta_{3}\right)^{-1} w_{2}, \hat{z}_{1}\right)\right) \\
&=\int_{U_{2}} \exp \left(-\pi \lambda^{2 n_{1}} w_{1} d \lambda^{2 n-2 n_{1}} w_{2}\right. \\
&\left.\cdot \int_{U_{1}} \exp \left(-\frac{\pi}{2} L_{\Phi_{p}}\left(\eta, \alpha_{1} ; w_{1}, \hat{z}_{1}\right)\right) d \lambda^{2 n} \bar{w}_{2}\right) d \lambda_{1}^{2 n-2 n_{1}} w_{2} \\
&= c_{1}\left(\beta_{3}\right) \int_{U_{1}} \exp \left(-\frac{\pi}{2} L_{\Phi_{p}}\left(\eta, \alpha_{1} ; w_{1}, \hat{z}_{1}\right)\right) d \lambda^{2 n_{1}} w_{1} .
\end{aligned}
$$

Substituting in (14), pulling out the $\widetilde{\beta}_{1}^{*}\left(\beta_{2}, \beta_{3}\right)$ term, and integrating over $\left(\Gamma_{p}^{*}\right)_{\alpha_{1}}$ $\times \Delta$ as in [1, proof of 2.11], we see that

$$
\hat{P}_{\Omega_{0}}\left(v ; \alpha_{1}, \hat{z}_{1}\right)=c_{2}\left(p, v_{2}, v_{3}\right) \hat{P}_{\Omega_{p}}\left(p ; \alpha_{1}, \hat{z}_{1}\right) .
$$

Since $1=\hat{P}(x ; 0,0)$ for all Poisson kernels, we have $C_{2} \equiv 1$. This completes the proof.

2.5. Theorem (Tight $C_{0}^{*}$ CONVERgence and $L^{q}$ boundary values). Suppose $P_{v}(x, z)=P(v ; x, z)$ is the Poisson kernel of $\Omega=\Omega_{\Gamma, \Phi} ; p$ is a regular point of $\bar{\Gamma}$ with respect to $\Phi$; and for $0<\delta \leqslant \delta_{0}>0, v_{\delta} \in \Gamma$ and $\lim _{\delta \rightarrow 0} v_{\delta}=$ $p$ admissibly $[1,2.9]$. Then $\lim _{\delta \rightarrow 0} P_{v_{\delta}}$ exists in the tight $C_{0}^{*}$ sense for 
measures on $N \approx \mathbf{R}^{m} \oplus \mathbf{C}^{n}$; and it equals the measure $d \mu_{p}$ which is defined for $g \in C_{0}(N)$ by

$$
\int g d \mu_{p}=\int_{T_{1} \oplus U_{1}} g\left(x_{1}, z_{1}\right) P\left(\Gamma_{p}, \Phi_{p} ; p ; x_{1}, z_{1}\right) d \lambda^{m_{1}} x_{1} d \lambda^{2 n_{1}} z_{1} .
$$

If $f \in L^{q}(N), 1 \leqslant q<\infty$, and we define the Poisson integral $P f$ of $f$ as in 1.5 , then under the above assumptions, in the $L^{q}$ norm,

$$
\begin{aligned}
\lim _{\delta \rightarrow 0} P f\left((x, z)\left[\left(i v_{\delta}, 0\right)\right]\right) & =P f((x, z)[(i p, 0)])=P f(p ; x, z) \\
& =\left(f * \mu_{p}\right)(x, z),
\end{aligned}
$$

where the middle equalities are definitions, and the right-hand convolution is in $N$ and well defined almost everywhere:

$$
\begin{aligned}
& \left(f * \mu_{p}\right)(x, z) \\
& \quad=\int_{T_{1} \oplus U_{1}} f\left((x, z) \circ\left(-t_{1},-w_{1}\right)\right) P\left(\Gamma_{p}, \Phi_{p} ; p ; t_{1}, w_{1}\right) d \lambda^{2 n_{1}} w_{1} d \lambda^{m_{1}} t_{1} .
\end{aligned}
$$

Proof. We note first that by the nature of the standard differentiator at $p$, and the kernel continuity theorem for $\Omega_{\delta}=\Omega_{\Gamma_{\delta}, \Phi_{\delta}}$, it follows that for arbitrary $\epsilon>0$ there exists $M<\infty, \delta_{1}>0$ such that if $\delta<\delta_{1}$ and

$$
\begin{aligned}
E(M, \epsilon)=\left\{\left(x_{1}+x_{2}+x_{3}, z_{1}+z_{2}\right): x_{j} \in T_{j}, z_{j} \in U_{j},\right. \\
\\
\left.\left|x_{1}\right|^{2}+\left|z_{1}\right|^{2} \leqslant M^{2},\left|x_{2}\right|^{2}+\left|x_{3}\right|^{2}+\left|z_{2}\right|^{2} \leqslant \epsilon^{2}\right\}
\end{aligned}
$$

then

$$
\int_{N-E(M, \epsilon)} P\left(v_{\delta} ; x, z\right) d \lambda^{m} x d \lambda^{2 n} z<\epsilon .
$$

Now by uniform continuity of $g$ on $E(M, \epsilon)$, and Lemma 2.4 , the first paragraph follows as in $[1,2.11]$. The second part now follows using $[1,1.8]$, which works just as well for $L^{q}(1<q<\infty)$.

III. Weak-type (1.1) boundedness and almost everywhere convergence. The polytopic case. We begin by considering octant-based Siegel domains: as in [1] these will prove the key to polytopic Siegel domains, or Siegel domains in which $\Gamma$ is a polytopic cone. Thus we assume $\Gamma=\Gamma_{+}^{m}=(0, \infty)^{m}$, and that each coordinate function of $\Phi$ is $\geqslant 0$. In the following, $l$ will denote a vector of $Z^{m}$, and $2^{l}$ the vector $\left(2^{l_{1}}, 2^{l_{2}}, \ldots, 2^{l_{m}}\right)$. We say $l \leqslant l^{\prime}$ if $l_{j} \leqslant l_{j}^{\prime} \forall j$.

$\sigma$ will denote a permutation of $(1, \ldots, m)$. We say $l$ belongs to $\sigma$ if $l_{\sigma_{1}} \geqslant l_{\sigma_{2}} \geqslant \cdots \geqslant l_{\sigma_{m}}$. Denoting by $\alpha^{j}$ the $j$ th canonical basis vector $\left(0, \ldots, 0,1_{j}\right.$, $0, \ldots, 0) \in \mathbf{R}^{m}$, we introduce for each $\sigma$ the unique orthogonal decomposition $\mathbf{C}^{n}=U_{1}^{\sigma} \oplus U_{2}^{o} \oplus \cdots \oplus U_{m}^{o}$ defined by 


$$
U_{1}^{\sigma} \oplus U_{2}^{\sigma} \oplus \cdots \oplus U_{j}^{\sigma}=\left\{z \in \mathbf{C}^{n}: \Phi(z) \in\left[\left\{\alpha^{\sigma}{ }^{\sigma}, \alpha^{\sigma_{2}}, \ldots, \alpha^{\sigma_{j}}\right\}\right]\right\} .
$$

By induction on $k=m-j+1$, using the positivity of the $\Phi\left(\alpha^{j}\right)$, we see as in 2.2 that in $\left(U_{1}^{\sigma}, \ldots, U_{m}^{\sigma}\right)$-block matrix form:

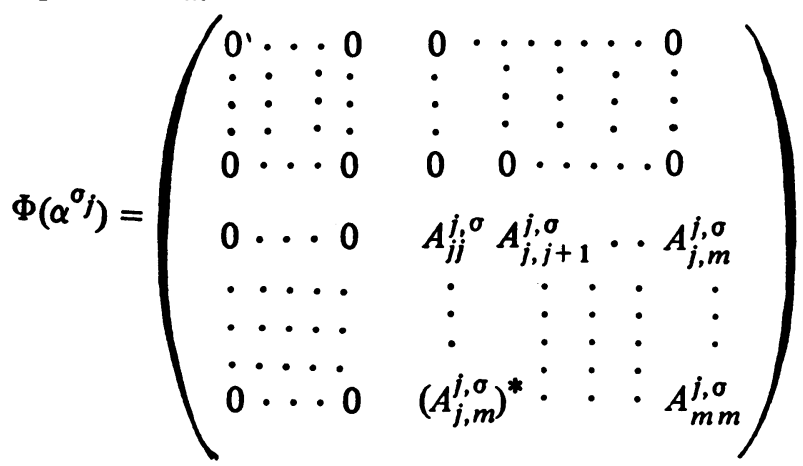

with $A_{j i}^{i, \sigma}>0$ for each $j, \sigma$ (unless $\operatorname{dim} U_{j}^{\sigma}=0$ ).

Now for $l$ belonging to $\sigma$ we set $r(l)=\sum_{j=1}^{m} l_{\sigma_{j}} \operatorname{dim} U_{j}^{\sigma}, s(l)=\sum_{j=1}^{m} l_{j}$; and we define the l-dilation $A_{l} \oplus B_{l}$ by setting

$$
A_{l}=\left(\begin{array}{cc}
2^{-l_{1}} & 0 \\
\cdot & 0 \\
0 & 2^{-l_{m}}
\end{array}\right) \text { and } B_{l}=\sum_{j=1}^{m} 2^{-l_{\sigma_{j}} / 2} \pi_{j}^{\sigma},
$$

where $\pi_{j}^{\sigma}$ is the orthogonal projection of $\mathbf{C}^{n}$ onto $U_{j}^{\sigma}$. We thus get $A_{l} \Gamma=\Gamma$, but $\Phi_{l}=A_{l} \Phi^{B} l$ is given in block matrix form by replacing the $A_{r s}^{j, \sigma}$ in (35) by $2^{\left(l_{\sigma_{r}}+l_{\sigma_{s}}-2 l_{\sigma_{j}}\right) / 2} A_{r s}^{j \sigma}$.

Note that if $l$ belongs to more than one permutation $\sigma$, then $r(l), s(l), A_{l}$, $B_{l}$, and $\Phi_{l}$ are independent of the choice of $\sigma$. Since for any $l$ and $j$, a $\sigma$ can be found such that $l$ and $l+\alpha^{j}$ both belong to $\sigma$, this implies $r(l)$ is a nondecreasing function of $l$; in fact, for $l \geqslant l_{0}$, we get $0 \leqslant r(l)-r\left(l_{0}\right) \leqslant n\left(s(l)-s\left(l_{0}\right)\right)=$ $n s\left(l-l_{0}\right)$.

3.1. Claim. For any compact $K \subset \Gamma$ there are constants $C_{1}(K), C_{2}(K)$ such that

$$
C_{1}(K) \leqslant \operatorname{det} \Phi_{l}(\alpha) \leqslant C_{2}(K) \quad \forall l \in Z^{m}, \alpha \in K
$$

Proof. It suffices to assume $l$ belongs to a fixed permutation $\sigma$. But since under this assumption $l_{\sigma_{r}}+l_{\sigma_{s}}-2 l_{\sigma_{j}} \leqslant 0$ for $r>j, s \geqslant j$, it follows by (35) and the following paragraph that $\left\{\Phi_{l}: l\right.$ belongs to $\left.\sigma\right\}$ is a precompact set. If $\alpha=$ $\left(\alpha_{1}, \ldots, \alpha_{m}\right) \in \Gamma$ it follows from (35), by successively clearing the rows and columns of $\Phi_{l}(\alpha)$ as in (23), that

$$
\operatorname{det} \Phi_{l}(\alpha) \geqslant \prod_{j=1}^{m}\left(\alpha_{\sigma_{j}}\right)^{\operatorname{dim} U_{j}^{\sigma} \operatorname{det} A_{j j}^{j, \sigma}>0}
$$


$\forall l$ belonging to $\sigma$; thus for $\alpha \in K$ we have $\operatorname{det} \Phi_{l}(\alpha) \geqslant C_{1}(\sigma, K)>0$. The existence of $C_{2}(\sigma, K) \geqslant \operatorname{det} \Phi_{l}(\alpha)$ follows by boundedness.

3.2. Definition. Suppose $v \in \Gamma=\Gamma_{+}^{m}$. For $l \in \mathbf{Z}^{m}$ let $E_{l}=E_{l}^{1} \oplus E_{l}^{2}$ where $E_{l}^{1}=\left\{\left(x_{1}, \ldots, x_{m}\right) \in \mathbf{R}^{m}:\left|x_{i}\right| \leqslant 2^{l_{i}}\right\}$ and $E_{l}^{2}=\left\{z \in \mathbf{C}^{n}:\left\langle\Phi(z), \alpha^{j}\right\rangle\right.$ $\left.\leqslant 2^{l_{j}} \forall j\right\}$. Define $l_{0}=l_{0}(v)=\left(l_{01}, l_{02}, \ldots, l_{l m}\right)$ where $2^{l_{0 j}-1}<v_{j} \leqslant 2^{l_{0 j}}$ each $j$. The $v$-partition of $\mathbf{R}^{m} \oplus \mathbf{C}^{n}$ is the set $\left\{E_{l}(v): l \geqslant l_{0}\right\}$ where

$$
\widetilde{E}_{l}(v)=E_{l}-\bigcup\left\{E_{l-\alpha} j: j \in\{1, \ldots, m\} \text { and } l-\alpha^{j} \geqslant l_{0}(v)\right\} \text {. }
$$

The scalar expression $z^{t} \Phi(\alpha) \bar{z}=\langle\Phi(z), \alpha\rangle$ will henceforth be denoted by $\Phi(\alpha)(z)$, for $z \in \mathbf{C}^{n}, \alpha \in \mathbf{R}^{m}$.

It is immediate that $\mathbf{R}^{m} \oplus \mathbf{C}^{n}$ is the disjoint union of the $\tilde{E}_{l}(v)$. We now estimate $\lambda^{m+2 n}\left(E_{l}\right)$ and $\max \left\{P(v ; x, z):(x, z) \in \widetilde{E}_{l}(v)\right\}$. This will enable us essentially to show $P_{v}$ is "radially almost-everywhere fast-decreasing" $[1,1.11$, $1.12]$, and thus to get the weak-type inequality.

\subsection{Lemma. There exist constants $c_{1}, c_{2}>0$ (dependent on $\left.\Phi\right)$ such} that

$$
2^{s(l)+r(l)} c_{1} \leqslant \lambda^{m+2 n}\left(E_{l}\right) \leqslant 2^{s(l)+r(l)} c_{2}
$$

$$
P\left(\Gamma_{+}^{m}, \Phi ; v ; x, z\right) \leqslant c_{2} \cdot 2^{-s(l)-r(l)} \cdot 2^{-\left[s(l)-s\left(l_{0}\right)+r(l)-r\left(l_{0}\right)\right]}
$$

for $l_{0}=l_{0}(v)$ and $(x, z) \in \widetilde{E}_{l}(v)$.

Proof. Obviously $\lambda^{m}\left(E_{l}^{1}\right)=2^{m} \cdot 2^{s(l)}$. If we define $F_{l} \subset \mathbf{C}^{n}$ by

$$
F_{l}=\left\{z \in \mathbf{C}^{n}: \Phi\left(2^{-l}\right)(z) \leqslant 1\right\},
$$

we easily see that $F_{l} \subset E_{l}^{2} \subset \sqrt{ } m F_{l}$. Diagonalizing $\Phi\left(2^{-l}\right)$ we note that $\lambda^{2 n}\left(F_{l}\right)=c / \operatorname{det} \Phi\left(2^{-l}\right)$, where $c=$ volume of unit ball. This proves (38), using (36) and the fact that $\operatorname{det} \Phi\left(2^{-l}\right)=2^{-r(l)} \operatorname{det} \Phi_{l}(1,1, \ldots, 1)$.

To prove (39) we get an explicit formula for the Szegö kernel. We have

$$
S(v ; x, z)=2^{n-m_{1}} \pi^{-m-n} \int_{0}^{\infty} e^{-\xi_{1} t_{1}} \int_{0}^{\infty} e^{-\xi_{2} t_{2}}
$$

$$
\cdots \int_{0}^{\infty} e^{-\zeta_{m} t_{m}} P_{\Phi}\left(t_{1} \cdots t_{m}\right) \cdot d t_{m} \cdots d t_{1}
$$

where $\zeta_{j}=v_{j}+\Phi\left(\alpha^{j}\right)(z)-i x_{j}$ and

$$
P_{\Phi}\left(t_{1}, \ldots, t_{m}\right)=\operatorname{det} \Phi\left(t_{1}, \ldots, t_{m}\right)
$$

If we define

$$
=\sum_{r_{1}+\cdots+r_{m}=n ; r_{j}>0} a_{r_{1} r_{2} \cdots r_{m}} t_{1}^{r_{1}} \cdots t_{m}^{r_{m}} .
$$




$$
\begin{aligned}
& Q_{\Phi}\left(t_{1}, \ldots, t_{m}\right)=\sum_{r_{1}+\cdots+r_{m}=n ; r_{j}>0}\left(r_{1} !\right)\left(r_{2} !\right) \cdots\left(r_{m} !\right) \\
& \cdot a_{r_{1} r_{2}} \cdots r_{m} t_{1}^{r_{1}} \cdots t_{m}^{r_{m}}
\end{aligned}
$$

we find after substituting in (41) and using the gamma function integral that

$$
S(v ; x, z)=2^{n-m} \pi^{-m-n} Q\left(\frac{1}{\zeta_{1}}, \frac{1}{\zeta_{2}}, \ldots, \frac{1}{\zeta_{m}}\right) . \prod_{j=1}^{m}\left(\frac{1}{\zeta_{j}}\right) .
$$

Since $\left\{\Phi_{l}: l \in Z^{m}\right\}$ is bounded this implies

$$
\begin{aligned}
& \left|S\left(\Gamma_{+}^{m}, \Phi_{l} ; v ; x, z\right)\right| \leqslant c(\epsilon) \quad \text { for all } l \in Z \\
& \left|v_{j}+\Phi_{l}\left(\alpha^{j}\right)(z)\right|^{2}+\left|x_{j}\right|^{2}=\left|\zeta_{j}\right|^{2} \geqslant \epsilon^{2}
\end{aligned}
$$

If $l_{0}=l_{0}(v)$ for some fixed $v, l \geqslant l_{0}$, and $(x, z) \in \widetilde{E}_{l}(v)$, we have for each $j \in$ $\{1, \ldots, m\}$ one of the three following: $\left|v_{j}\right| \geqslant 2^{l_{j}-1}, \Phi\left(\alpha^{j}\right)(z) \geqslant 2^{l_{j}-1}$, or $\left|x_{j}\right| \geqslant 2^{l_{j}-1}$. That is, $\left|\left(A_{l} v\right)_{j}\right| \geqslant 1 / 2,\left|\Phi_{l}\left(\alpha^{j}\right)\left(B_{l} z\right)\right| \geqslant 1 / 2$, or $\left|\left(A_{l} x\right)_{j}\right| \geqslant 1 / 2$. Hence by (45) and (10),

$$
\begin{aligned}
\left|S\left(\Gamma_{+}^{m}, \Phi ; v ; x, z\right)\right| & =2^{-s(l)-r(l)}\left|S\left(\Gamma_{+}^{m}, \Phi_{l} ; A_{l} v ; A_{l} x, B_{l} z\right)\right| \\
& \leqslant c \cdot 2^{-s(l)-r(l)} .
\end{aligned}
$$

But since the closure of $\left\{\Phi_{l}: l \in Z^{m}\right\}$ in $\Theta$ consists by (36) only of $\Gamma_{+}^{m}$-definite forms, the kernel continuity theorem implies the positive function $S\left(\Gamma_{+}^{m}, \Phi ; v ; 0,0\right)$ is $\geqslant c_{1}>0$ for $\Phi=\Phi_{l}, l \in Z^{m}$, and $1 \leqslant \tilde{v}_{j} \leqslant 2, \forall j$. Setting $\tilde{v}=2 A_{l_{0}} v$ we get (39) using (8).

3.4. Definition. For $l \in Z$ define $\Psi_{l}(x, z)=\left(\lambda^{2 n+m}\left(E_{l}\right)\right)^{-1} \chi_{E_{l}}(x, z)$. Suppose $D \subset\{1,2, \ldots, m\}$. We define $l_{k}=l_{k}(D)=k \Sigma_{j \in D} \alpha^{j}, T_{D}=$ $\left\{x \in \mathbf{R}^{m}: x_{j}=0 \forall j \in D\right\}, \Gamma_{D}=\operatorname{Inn}\left(T_{D} \cap \bar{\Gamma}_{+}^{m}\right), U_{D}=\left\{z \in \mathbf{C}^{n}: \Phi(z) \in T_{D}\right\}$. $\pi_{D}^{1}: \mathbf{C}^{n} \rightarrow U_{D}^{1}$ and $\tau_{D}: \mathbf{R}^{m} \rightarrow T_{D}$ are the orthogonal projections. The admissible region $R_{\epsilon} \subset \Gamma_{+}^{m} \oplus \mathrm{R}^{m} \oplus \mathrm{C}^{n}$ is defined for $\epsilon>0$ by

$$
R_{\epsilon}=R_{\epsilon}(D)=\left\{(v ; x, z): v_{j} \geqslant \epsilon\left|\tau_{D}^{1} v\right| \forall j \in D ;\right.
$$

$$
\left.\epsilon\left|\tau_{D}^{1} x\right| \leqslant\left|\tau_{D}^{1} v\right|, \epsilon\left|\pi_{D}^{\frac{1}{2}} z\right|^{2} \leqslant\left|\tau_{D}^{\frac{1}{D}} v\right|\right\} .
$$

Lastly, for every integer $k$ we define the $k$-majorant

$$
H_{D, k}=\sum_{l>0} 2^{-s(l)} \Psi_{l_{k}+l}
$$

3.5. THEOREM (ALMOST EVERYWHERE LIMITS). SUppose $\Omega=\Omega_{\Gamma, \Phi}$ is a polytopic Siegel domain of class $(m, n)$ and suppose $p \in \bar{\Gamma}, T=\left[\Gamma_{p}\right], U=$ $\left\{z \in \mathbf{C}^{n}: \Phi(z) \in T\right\}$. Let $\pi: \mathbf{C}^{n} \rightarrow U, \tau: \mathbf{R}^{m} \rightarrow T$ be the orthogonal pro- 
jections, $\pi^{\perp}=I-\pi, \tau^{\perp}=I-\tau$. Let $\Lambda=\pi^{\perp} \Gamma \subset T^{\perp}$, and define

$$
\begin{aligned}
R_{\epsilon}^{2}=\left\{\left(v^{2} ; x^{2}, z^{2}\right) \in \Lambda \oplus T^{\perp} \oplus U^{\perp}: \operatorname{dist}\left(v^{2}, \partial \Lambda\right) \geqslant \epsilon\left|v^{2}\right| ;\right. \\
\left.\epsilon\left|x^{2}\right| \leqslant\left|v^{2}\right|, \epsilon\left|z^{2}\right|^{2} \leqslant\left|v^{2}\right|\right\} .
\end{aligned}
$$

Suppose $f \in L^{1}\left(\mathbf{R}^{m} \oplus \mathbf{C}^{n}\right)$. Then for almost every $\left(x^{2}, z^{2}\right) \in T^{\perp} \oplus U^{\perp}$ the following holds: Let $K \subset \Gamma_{p} \oplus T \oplus U$ be compact, and $\epsilon>0$. If for $0<\delta<$ $\delta_{0}>0,\left(v_{\delta}^{2} ; x_{\delta}^{2}, z_{\delta}^{2}\right) \in R_{\epsilon}^{2}$ and $\lim _{\delta \rightarrow 0}\left(v_{\delta}^{2} ; x_{\delta}^{2}, z_{\delta}^{2}\right)=0$, then (with definitions as in 2.5)

$$
\begin{aligned}
\lim _{\delta \rightarrow 0} P f\left(v^{1}+v_{\delta}^{2} ;\left(x^{1}+x^{2}, z^{1}+z^{2}\right) \circ\left(x_{\delta}^{2}, z_{\delta}^{2}\right)\right) \\
=P f\left(v^{1} ; x^{1}+x^{2}, z^{1}+z^{2}\right)
\end{aligned}
$$

uniformly over $\left(v^{1} ; x^{1}, z^{1}\right) \in K$.

Proof. To begin with we assume $\Gamma=\Gamma_{+}^{m}$. Note that $l \leqslant l^{\prime} \Rightarrow E_{l} \subset E_{l^{\prime}}$. Moreover, the Schwarz inequality on the quadratic forms $\Phi\left(\alpha^{j}\right)(z)=\left\langle\Phi(z), \alpha^{j}\right\rangle$ implies $E_{l} \circ E_{l} \subset 4 E_{l}$ and $E_{l} \circ E_{l} \circ E_{l} \subset 9 E_{l}$. Since $E_{l}$ is symmetric in $N$, it follows by a slight variation in the proof of Lemma 2.2 of [5] that if $k \mapsto l_{k}^{\prime}$ is any increasing function from $\mathbf{Z}$ into $\mathbf{Z}^{m}$, then

(51) $\lambda^{m+2 n}\left(\left\{\nu \in N:\left(f * \Psi_{l_{k}^{\prime}}\right)(\nu) \geqslant t\right.\right.$ some $\left.\left.k \in Z\right\}\right) \leqslant c\|f\|_{1} / t \quad \forall t>0$.

Lemma 2.3 of [5] implies that a similar weak-type inequality holds when one replaces $\Psi_{l_{k}^{\prime}}$ with $H_{D, k}$ in (51).

We claim that if $\epsilon>0, K \subset \Gamma_{p} \oplus T \oplus U$ is compact, $(v ; x, z) \in R_{\epsilon} \cap$ $(\tau \oplus \tau \oplus \pi)^{-1} K, k$ is the integer such that $2^{k-1}<\left|\tau^{\perp} v\right| \leqslant 2^{k}$, and $D=$ $\left\{j \in\{1, \ldots, m\}: p_{j}=0\right\}$, then

$$
P(v ;(t, w) \circ(x, z)) \leqslant c(\epsilon, K) H_{D, k}(t, w),
$$

where $c(\epsilon, K)$ is independent of $k$. Indeed, the conditions imply there exists $\tilde{l}>0$ dependent only on $K$ and $\epsilon$, such that $l_{k}-\tilde{l} \leqslant l_{0}(v) \leqslant l_{k}+\tilde{l}$ and $(x, z) \epsilon$ $E_{l_{k}+2 \tilde{l}}$. Also we may assume $E_{l} \circ E_{l} \subset E_{l+\tilde{l}}$ for all $l$. The claim now follows from Lemma 3.3, and the definitions, since $\Psi_{l} \leqslant c(\tilde{l}) \Psi_{l+\tilde{l}}$ independently of $l$.

For $f \in L^{1}$, choose for each integer $r>0$ a function $h_{r} \in L^{1}$ such that $\left\|h_{r}\right\|_{1} \leqslant 2^{-r}$ and $f-h_{r} \in C_{0}$. Define the regular set $Q \subset N$ as follows:

$$
Q=\left\{(x, z): \limsup _{r \rightarrow \infty}\left[\sup _{k \in \mathbf{Z}}\left(\left|h_{r}\right| * H_{D, k}\right)(x, z)\right]=0\right\},
$$

with convolution in $N$. If $L \subset T \oplus U$ is compact there exists $l^{\prime}=l^{\prime}(L) \in \mathrm{Z}^{m}$ such that $L \subset E_{l^{\prime}+l_{k}} \forall k \in Z$. It follows that for $\left(x^{1}, z^{1}\right) \in L$,

$$
H_{D, k}\left[(t, w) \circ\left(x^{1}, z^{1}\right)\right] \leqslant c(L) H_{D, k}(t, w)
$$

independently of $k$. Therefore if $(x, z) \in Q$, 


$$
\lim _{r \rightarrow \infty}\left(\sup _{k \in Z}\left(\left|h_{r}\right| * H_{D, k}\right)\left[(x, z) \circ\left(x^{1}, z^{1}\right)\right]\right)=0
$$

uniformly over $\left(x^{1}, z^{1}\right) \in L$ for any compact $L \subset T \oplus U$.

We now generalize to $\Gamma$ polytopic. In this case, $\bar{\Gamma}^{*}$ can be written as the almost disjoint union of linear transforms of $\bar{\Gamma}_{+}^{m}$. Summing a finite number of transforms of $H_{D, k}$, we get by (17) of [1] a majorant $H_{\Gamma ; D, k}$ for $P_{\Gamma, \Phi}$ which is easily shown to satisfy (51)-(55). Indeed, if $\bar{\Gamma}^{*}=\bigcup_{j} \bar{\Gamma}_{j}^{*}$ is the almost disjoint union, then $\Gamma_{j p} \supset \Gamma_{p} \forall j$, and minor manipulations with linear transformations give the results (cf. proof of $[1,3.7]$ ).

Since $\Gamma$ is polytopic, $r(p)=0$ and hence $p$ is a regular point of $\bar{\Gamma}$ with respect to $\Phi$ (see 4.4 below). Thus for each $r>0$, the theorem holds for $f-h_{r}$ $\in C_{0}$ by 2.5. But by (55) we may express $Q=Q^{2} \circ(T \oplus U)=Q^{2}+(T \oplus U)$ where $Q^{2} \subset T^{\perp} \oplus U^{\perp}$. By the usual arguments (see proof of $[1,1.14]$ ), it follows from (55) that (50) holds uniformly for $\left(v^{1} ; x^{1}, z^{1}\right) \in K$ if $(x, z)=$ $\left(x^{2}, z^{2}\right) \in Q^{2}$; the skewing caused by the o-product makes no essential difference. But $N-Q$ has measure 0 which implies $\left(T^{\perp} \oplus U^{1}\right)-Q^{2}$ has $\left(T^{\perp} \oplus U^{1}\right)$-measure 0 . This completes the proof.

3.6. Corollary. Theorem 3.6 holds where the left-hand term in (50) is replaced by

$$
\lim _{\delta \rightarrow 0} P f\left(v^{1}+v_{\delta}^{2} ; x^{1}+x^{2}+x_{\delta}^{2}, z^{1}+z^{2}+z_{\delta}^{2}\right),
$$

if the set $R_{\epsilon}^{2}$ in the hypothesis is replaced by

$$
\begin{aligned}
\widetilde{R}_{\epsilon}^{2}=\left\{\left(v^{2} ; x^{2}, z^{2}\right) \in \Lambda \oplus T^{1} \oplus U^{1}:\right. & \operatorname{dist}\left(v^{2}, \partial \Lambda\right) \geqslant \epsilon\left|v^{2}\right| ; \\
& \left.\epsilon\left|x^{2}\right| \leqslant\left|v^{2}\right|, \epsilon\left|z^{2}\right| \leqslant\left|v^{2}\right|\right\} .
\end{aligned}
$$

Proof. Note that the last inequality in the definition makes $\widetilde{R}_{\epsilon}^{2}$ a narrower set. The proof follows by converting the argument of $P f$ into the form of (50).

IV. Examples, remarks, conjectures. In a Siegel domain $\Omega=\Omega_{\Gamma, \Phi}$ we have $\Phi\left(C^{n}\right) \subset \bar{\Gamma} \subset R^{m}$, where $\Phi(z)=\Phi(z, z), z \in C^{n}$. The image set $\Phi\left(C^{n}\right)$ is a cone, but it may not be convex; it is therefore convenient to deal with $\bar{\Phi}=$ closed convex hull of $\Phi\left(C^{n}\right)$. (For instance in $\Omega_{I(2,3)}$ of 4.2 below, $\Phi\left(C_{1,2}\right)=$ $\partial \bar{\Phi}$.)

We will call a closed convex cone $\bar{\Delta}$ thick if $\Delta=\operatorname{Inn} \bar{\Delta}$ is open, or equivalently if $\bar{\Delta}$ has nonempty interior. $\bar{\Delta}$ will be said to be narrow if $\bar{\Delta}$ contains no whole line. Clearly these conditions are dual, and $\Delta$ is proper if and only if it is thick and narrow. (Here we apply the same terms to $\Delta=\operatorname{Inn} \bar{\Delta}$ as to $\bar{\Delta}$.)

Since $\bar{\Phi}$ is narrow, its dual cone $\bar{\Phi}^{*}$ is thick. In fact

$$
\bar{\Phi}^{*}=\left\{\alpha \in \mathbf{R}^{m}: \Phi(\alpha) \geqslant 0\right\},
$$


where $\alpha \mapsto \Phi(\alpha) \in H_{n}=\{n \times n$ Hermitian matrices $\}$ is as defined in 1.4.

Since $\Phi(\alpha)$ and $\Phi(-\alpha)$ are both $\geqslant 0$ if and only if $\Phi(\alpha)=0$, we see that $\bar{\Phi}^{*}=T_{0} \oplus \bar{\Delta}^{*}$, where $T_{0}=\left\{\alpha \in R^{m}: \Phi(\alpha)=0\right\}$ and $\bar{\Delta}^{*}$ is a proper cone of $T_{0}^{1}$. Thus $\bar{\Phi}^{*}$, and hence $\bar{\Phi}$, are proper if and only if $\alpha \mapsto \Phi(\alpha)$ is $1-1$.

4.1. Definitions. (a) Suppose $m \geqslant 0$, and $\Gamma$ is a proper cone of $\mathbf{R}^{m}$. We call $\Gamma$ a Hermitian cone if either $m=0$, or there exists $l>0$ and a 1-1 linear map $\psi: \mathbf{R}^{m} \rightarrow H_{l}$ such that $\Gamma=\left\{\alpha \in \mathbf{R}^{m}: \psi(\alpha)>0\right\}$. $\Gamma$ is a Hermitian dual cone if $\Gamma^{*}$ is a Hermitian cone.

(b) Suppose $\Omega=\Omega_{\Gamma, \Phi}$ is a Siegel domain. We call $\Omega$ minimal (with respect to $\Phi)$ if $\bar{\Phi}=\bar{\Gamma}$, and loose if $\bar{\Phi}-\{0\} \subset \Gamma$.

We note at once that in the decomposition $\bar{\Phi}^{*}=T_{0} \oplus \bar{\Delta}^{*}, \Delta^{*}$ is a Hermitian cone; this follows from the $\Gamma$-definiteness of $\Phi$. Thus $\bar{\Phi}$ is a Hermitian dual cone on the subspace $[\bar{\Phi}]$. Note also that $4.1(\mathrm{~b})$ can be expressed in dual terms: $\Omega$ is minimal iff $\bar{\Phi}^{*}=\bar{\Gamma}^{*}$, and loose iff $\bar{\Gamma}^{*}-\{0\} \subset \Phi^{*}=\operatorname{Int} \bar{\Phi}^{*}$. The "minimal" and "loose" Siegel domains are extreme cases, as can be seen by consulting the definition; but in fact most common examples of Siegel domains fall into one or the other of these categories. Tube domains are examples of loose Siegel domains; the common nontube infinite realizations of Cartan domains are all minimal Siegel domains.

4.2. EXAmples. The best known examples of Siegel domains are the infinite realizations of Cartan domains [3] , [4], [5]. The simplest of these is the type I domain of order $(k, k+l), k>0, l \geqslant 0$. Here $m=k^{2}, n=k l$, and if we identify $\mathbf{R}^{m}$ with $H_{k}$ using as inner product in $H_{k}+i H_{k}=\mathrm{C}_{k}$ the form $\langle A, B\rangle=\operatorname{tr} A B^{*}$, then the Siegel domain $\Omega_{\Gamma(k+l, k)}=\Omega$ is $\Omega_{\Gamma, \Phi}$, where $\Gamma=$ $H_{K}^{+}, \mathrm{C}^{n}=\mathrm{C}_{k l}=\{k \times l$ complex matrices $\}$ with inner product $\langle E, F\rangle=$ $\operatorname{tr} E F^{*}=\operatorname{tr} F^{*} E$, and

$$
\Phi(E, F)=E F^{*} \subset \mathrm{C}_{k} \text {. }
$$

ExAMPLE 4.2(a). If $l>0$ clearly $\bar{\Phi} \subset \bar{\Gamma}$. But $\bar{\Phi}^{*}=\left\{B \in H_{k}:\right.$ tr $E E^{*} B \geqslant$ $0 \forall E \in \mathrm{C}_{k l}$ \}. If $B \ngtr 0$ there exists unitary $U \in \mathrm{C}_{n}$ such that $U^{*} B U$ is real diagonal with negative upper left-hand corner entry. If

and $E=U E_{1}$ then

$$
E_{1}=\left(\begin{array}{cccc}
1 & 0 & \cdots & 0 \\
0 & 0 & \cdots & 0 \\
\vdots & \vdots & \ddots & \vdots \\
0 & 0 & \cdots & 0
\end{array}\right)
$$

(59) $\operatorname{tr} E E^{*} B=\operatorname{tr} U^{*}\left(E E^{*} B\right) U=\operatorname{tr} U^{*} E E^{*} U U^{*} B U=\operatorname{tr} E_{1} E_{1}^{*} U^{*} B U<0$.

Thus $\bar{\Phi}^{*} \subset \bar{H}_{k}^{+}, \bar{\Phi}^{*}=\bar{H}_{k}^{+}$, and $\Omega$ is minimal. 
Suppose $p \in \bar{H}_{k}^{+}$, rank $p=k_{1} \leqslant k$. There exists unitary $U \in \mathrm{C}_{k}$ such that $U^{*} p U$ is diagonal with the first $k_{1}$ elements on the diagonal all positive. Since $(A, E) \mapsto\left(U^{*} A U, U^{*} E\right)$ is an identification of $\Omega$ with itself (see 1.1), we may assume $p$ is of this form. Written in $\left(k_{1}, k-k_{1}\right)$-block matrix form, we then get for $k_{2}=k-k_{1}$,

$$
\begin{array}{cc}
T_{1}(p)=\left\{\left(\begin{array}{ll}
A_{1} & 0 \\
0 & 0
\end{array}\right): A_{1} \in H_{k_{1}}\right\}, & T_{2}(p)=\left\{\left(\begin{array}{cc}
0 & B \\
B^{*} & 0
\end{array}\right): B \in \mathrm{C}_{k_{1}, k_{2}}\right\}, \\
{ }_{(60)} T_{3}(p)=\left\{\left(\begin{array}{ll}
0 & 0 \\
0 & C
\end{array}\right): C \in H_{k_{2}}\right\}, & \Gamma_{p}=\left\{\left(\begin{array}{cc}
A_{1} & 0 \\
0 & 0
\end{array}\right): A_{1} \in H_{k_{1}}^{+}\right\}, \\
\Gamma^{*}(p)=\left\{\left(\begin{array}{ll}
0 & 0 \\
0 & C
\end{array}\right): C \in H_{k_{2}}^{+}\right\}, &
\end{array}
$$

where $H_{0}^{+}$is defined to equal $\{0\}$. The standard differentiator $A_{\delta} \oplus B_{\delta}$ acts on $\bigvee_{H_{k}} \oplus \mathrm{C}_{k l}$ as follows:

$$
\left(A_{\delta} \oplus B_{\delta}\right)(A, E)=\left(H_{\delta} A H_{\delta}, H_{\delta} E\right), \quad H_{\delta}=\left(\begin{array}{cc}
I & 0 \\
0 & \delta^{-1} I
\end{array}\right) .
$$

Thus $\Omega_{\delta}=\Omega, \Omega_{0}=\Omega$, and it is straightforward to show that $p$ is regular. If we write

$$
E=\left(\begin{array}{l}
E_{1} \\
E_{2}
\end{array}\right), \quad E_{1} \in \mathrm{C}_{k_{1}, l}, E_{2} \in \mathrm{C}_{k_{2}, l}
$$

then

$$
U_{1}=\left\{\left(\begin{array}{c}
E_{1} \\
0
\end{array}\right)\right\}, \quad U_{2}=\left\{\left(\begin{array}{c}
0 \\
E_{2}
\end{array}\right)\right\}, \quad \text { and } \quad \Omega_{p} \approx \Omega_{I\left(k_{1}, k_{1}+l\right)}
$$

in the obvious fashion, unless $k_{1}=0$ in which case $\Omega_{p}$ is trivial.

A similar analysis shows each point of the Siegel domain of type $\operatorname{IVb}(k)$ is regular: one merely has to shift over to quaternion-Hermitian matrices and quaternion inner products [5]. Again, the lower-dimensional Siegel domains of Theorem 2.5 are also of type $\mathrm{IVb}$, and the domain itself is minimal.

EXAMPLE 4.2(b). The classical tube domains all satisfy the hypotheses of Theorem 2.5. Indeed, the cones are the cones of positive definite Hermitian (type I), real symmetric (type II), and quaternion Hermitian (type IV(a)) matrices, respectively, all of which are regular cones in the sense that all their boundary points are regular. In each case the analogue of $(60)$ holds, implying that the lower-dimensional cones of [1, Theorem 2.11], are just lower-dimensional classical domains of the same type. The type III domain is just the light-cone tube domain, one of the "rounded" tube domains of [1].

REMARK. We note here that the direct sum of regular Siegel domains is regular (see 4.4 below). Thus all symmetric Siegel domains constructed from the classical domains are regular. The exceptional Siegel domains [3], [5] are pre- 
sumably regular also, although we have not carried out the calculations to prove this.

4.3. EXAmples. If we restrict ourselves to minimal Siegel domains, it is easy to construct "pathological" examples. They suggest that Hermitian dual cones might be an interesting object of study.

4.3(a). Define $\Phi: \mathbf{C}^{3} \times \mathrm{C}^{3} \rightarrow \mathrm{R}^{4}+i \mathrm{R}^{4}$ as follows:

$$
\Phi(z, w)=\left(z_{1} \bar{w}_{1}, z_{1} \bar{w}_{3}+z_{2} \bar{w}_{2}+z_{3} \bar{w}_{1}, z_{2} \bar{w}_{3}+z_{3} \bar{w}_{2}, z_{3} \bar{w}_{3}\right)
$$

Equivalently, $\Phi$ is the form such that

$$
\Phi(\alpha)=\left(\begin{array}{ccc}
\alpha_{1} & 0 & \alpha_{2} \\
0 & \alpha_{2} & \alpha_{3} \\
\alpha_{2} & \alpha_{3} & \alpha_{4}
\end{array}\right), \quad \alpha=\left(\alpha_{1}, \alpha_{2}, \alpha_{3}, \alpha_{4}\right) \in \mathbf{R}^{4}
$$

Since $\Phi[(1, \delta, 0,1)]>0$ for $0<\delta<1$, and since the $\Phi\left(\alpha^{j}\right)$ are linearly independent (for $\left\{\alpha^{j}\right\}$ the canonical basis of $\mathbf{R}^{4}$ ), $\bar{\Phi}$ and $\bar{\Phi}^{*}$ are proper cones. Let $\bar{\Gamma}=\bar{\Phi}$. Clearly $\alpha \in \bar{\Gamma}^{*} \Rightarrow \alpha_{1} \geqslant 0$ and $(0,0,0,1) \in \bar{\Gamma}^{*}$; hence $(1,0,0,0) \in$ $\partial \Gamma^{* *}=\partial \Gamma$. If $p=(1,0,0,0)$ it is easy to verify that $\Gamma^{*}(p)=\left\{\left(0,0,0, \alpha_{4}\right)\right.$ : $\left.\alpha_{4}>0\right\}$. But $U_{1}=\operatorname{Ker} \Phi[(0,0,0,1)]=\left\{z \in C^{3}: z_{3}=0\right\}$, so $\Phi\left(U_{1}\right)=$ $\left\{\left(\alpha_{1}, \alpha_{2}, 0,0\right): \alpha_{1}, \alpha_{2} \geqslant 0\right\}$, and $p$ is not "nice" with respect to $\Phi$ in the sense of 2.1 .

Since $\inf \left\{|\Phi(z)|: z \in \mathbf{C}^{3},|z|=1\right\}>0$, a compactness argument implies $\Phi\left(C^{3}\right)$ is closed and $\bar{\Phi}=$ convex hull of $\Phi\left(C^{3}\right)$. Hence $\bar{\Gamma}_{p} \subset$ (convex hull of $\left.\Phi\left(C^{3}\right)\right) \cap\left\{\alpha \in R^{4}: \alpha_{4}=0\right\}=\Phi\left(U_{1}\right)$. In fact $\bar{\Gamma}_{p}$ is the closed convex component of $p$ in $\Phi\left(U_{1}\right)$, hence $\bar{\Gamma}_{p}=\left\{\left(\alpha_{1}, 0,0,0\right): \alpha_{1} \geqslant 0\right\}$. Applying the standard differentiator $A_{\delta}$ of 2.2 to $\bar{\Gamma}=\bar{\Phi}$ we see that $A_{\delta} \bar{\Gamma}=\bar{\Phi}_{\delta}$ where $\Phi_{\delta}=A_{\delta} \Phi^{B}$ for a suitable $B_{\delta}$ :

$$
\Phi_{\delta}\left[\left(\alpha_{1}, \alpha_{2}, \alpha_{3}, \alpha_{4}\right)\right]=\left(\begin{array}{ccc}
\alpha_{1} & 0 & \alpha_{2} \\
0 & \alpha_{2} & \delta^{1 / 2} \alpha_{3} \\
\alpha_{2} & \delta^{1 / 2} \alpha_{3} & \alpha_{4}
\end{array}\right)
$$

Hence $\lim _{\delta \downarrow 0} A_{\delta} \bar{\Gamma}$ exists and equals $\bar{\Phi}_{0}$, where $\Phi_{0}=\lim _{\delta \downarrow 0} \Phi_{\delta}$. But $\Phi_{0}\left[\left(0,0, \alpha_{3}, 0\right)\right]=0$ for all $\alpha_{3} \in R$; hence $\bar{\Phi}_{0} \subset\left\{\alpha \in R^{4}: \alpha_{3}=0\right\}$ and is not proper. Thus we have an example of a Hermitian dual cone with a boundary point that is not "nice" even in the sense of $[1,2.6]$.

REMARK. The above example suggests that the definition of "nice" point be generalized to allow differentiators of the form

$$
A_{\delta}=\pi_{0}+\delta^{-1} \pi_{1}+\delta^{-r_{2}} \pi_{2}+\cdots+\delta^{-r_{k}} \pi_{k},
$$

where $1<r_{2}<\cdots<r_{k}$, the $\pi_{j}$ are pairwise orthogonal Hermitian projections with 
sum 1 , and $\pi_{0}\left(\mathbf{R}^{m}\right)=\left[\Gamma_{p}\right]$. It can be shown that if $\Gamma$ is a Hermitian dual cone, all points of $\bar{\Gamma}$ are "nice" under this definition. (The corresponding result for a Hermitian cone is an easy consequence of the result for $H_{n}^{+}$.)

Example 4.3(b). Let $\Phi: \mathbf{C}^{3} \times \mathbf{C}^{3} \rightarrow \mathbf{R}^{4}+i \mathbf{R}^{4}$ be defined as in (64) by

$$
\Phi(\alpha)=\left(\begin{array}{ccc}
\alpha_{1} & 0 & \alpha_{3} \\
0 & \alpha_{2} & \alpha_{3} \\
\alpha_{3} & \alpha_{3} & \alpha_{4}
\end{array}\right) \text { for } \alpha=\left(\alpha_{1}, \alpha_{2}, \alpha_{3}, \alpha_{4}\right) \in \mathbf{R}^{4}
$$

If we set $U_{1}=\left\{z \in \mathbf{C}^{3}: z_{3}=0\right\}$ we see that $\Phi\left(U_{1}\right)=\left\{\left(\alpha_{1}, \alpha_{2}, 0,0\right): \alpha_{1}, \alpha_{2} \geqslant\right.$ $0\}$. But $U_{1}=\operatorname{Ker} \Phi[(0,0,0,1)]$ and $(0,0,0,1) \in \partial \Phi^{*}$; hence choosing $p=$ $(1,1,0,0)$ we have $\bar{\Gamma}_{p}=\Phi\left(U_{1}\right), \Gamma^{*}(p)=\left\{\left(0,0,0, \alpha_{4}\right): \alpha_{4}>0\right\}$, and $U_{1}(p)=$ $U_{1}$.

By the construction we see that $p$ is a "nice" point of $\bar{\Gamma}=\bar{\Phi}$ with respect to $\Phi$; in fact, $\Gamma_{\delta}=\Gamma$ and $\Phi_{\delta}=A_{\delta} \Phi^{E_{\delta}}=\Phi$ for all $\delta>0$, since $\Phi$ is already in the form of (22). Hence $\Gamma_{0}^{*}=\Gamma^{*}=\left\{\alpha \in \mathbf{R}^{4}: \Phi(\alpha)>0\right\}$. Using this explicit expression, it is easy to check that $p$ is not a regular point of $\bar{\Phi}=\bar{\Gamma}$, even in the weak sense of $[1,2.10]$.

The strong regularity condition of 2.3 above is, of course, much harder to satisfy; a "random" choice is unlikely to satisfy $2.3(\mathrm{c})$. However, there are several common hypotheses which insure that this condition holds (besides the symmetry hypothesis of Example 4.2):

4.4. Claim. $p$ is a regular point of $\bar{\Gamma}$ with respect to $\Phi$ if any of the following hold:

(a) $p=0$ or $p \in \Gamma$;

(b) $p=A q, \Gamma=A \Delta$, and $\Phi=A \Psi^{B}$, where $A, B$ are nonsingular as in 1.3, and $q$ is regular in $\bar{\Delta}$ with respect to $\psi$;

(c) $p_{j}$ is a regular point of $\bar{\Gamma}_{j}$ with respect to $\Phi_{j}$; where $\Phi_{j}: \mathbf{C}^{n_{j}} \times \mathbf{C}^{n_{j}} \rightarrow$ $\mathbf{R}^{m_{j}}+i \mathbf{R}^{m_{j}}$ is $\Gamma_{j}$-definite, $\mathbf{R}^{m}=\mathbf{R}^{m_{1}} \oplus \mathbf{R}^{m_{2}}, \mathbf{C}^{n}=\mathbf{C}^{n_{1}} \oplus \mathbf{C}^{n_{2}}, \bar{\Gamma}=\bar{\Gamma}_{1} \oplus \bar{\Gamma}_{2}$, $\Phi=\Phi_{1} \oplus \Phi_{2}$, and $p=\left(p_{1}, p_{2}\right)$;

(d) $p$ is regular in the sense of $[1,2.10]$, and $p \notin \bar{\Phi}$;

(e) $r(p)=0$, that is, $\operatorname{dim}\left[\Gamma_{p}\right]+\operatorname{dim}\left[\Gamma^{*}(p)\right]=m$;

(f) $0 \neq p \in \partial \Gamma, k^{*}(p)=\operatorname{dim}\left[\Gamma^{*}(p)\right]=1, p$ is "nice" in the sense of $[1,2.6]$, and for any $\alpha_{0} \in \Gamma^{*}(p), 0<\operatorname{dim} \operatorname{Ker} \Phi(\alpha) \equiv$ constant for $\alpha \in \partial \Gamma^{*} \cap$ $N\left(\alpha_{0}\right)$, where $N\left(\alpha_{0}\right)$ is some neighbourhood of $\alpha_{0}$.

PRoof. (a) is trivial and (c) is straightforward from the definitions. (b) follows, as in [1, 2.13], by identifying (Definition 1.1) $A$ and $B$ with upper triangular matrices (see $[1,2.7])$, writing $\left(A_{\delta} \oplus B_{\delta}\right)(A \oplus B)=\left(\widetilde{A}_{\delta} \oplus \widetilde{B}_{\delta}\right) \cdot$ $\left(A_{\delta} \oplus B_{\delta}\right.$ ), and taking limits. (d) follows because 2.1(b) and 2.3(c) are both trivial consequences of the fact that $\operatorname{Ker} \Phi(\alpha)=0$ for $\alpha \in \Gamma^{*}(p)$. Points satisfying (e) are automatically "nice", by the remark following 2.1 , and $\bar{\Gamma}_{0}^{*}=\bar{\Gamma}_{p}^{*} \oplus$ 
$\bar{\Gamma}^{*}(p)$; since $B(\alpha)=0$ in $2.3(\mathrm{c})$ the claim follows in this case.

To prove (f), let $\rho: \mathbf{R}^{m-1} \rightarrow \mathbf{R}^{m}$ be an affine map with $\rho(0)=\alpha_{0}$ and $|\rho(\beta)| \geqslant\left|\alpha_{0}\right|$ for $\beta \in \mathbf{R}^{m-1}$. Without loss of generality we may assume that there are neighbourhoods $\tilde{N}_{j}$ of 0 in $\mathbf{R}^{m-j}(j=1,2)$ and a real valued function $\beta_{1}$ on $\widetilde{N}_{2}$ such that for $\beta^{\perp}=\left(\beta_{2}, \ldots, \beta_{m-1}\right), \beta_{1}\left(\beta^{\perp}\right) \geqslant 0$ and

$$
\left(\beta_{1}, \beta^{\perp}\right) \in \tilde{N}_{1} \cap \rho^{-1}\left(\partial \Gamma^{*}\right) \Longleftrightarrow \beta^{\perp} \in \tilde{N}_{2} \text { and } \beta_{1}=\beta_{1}\left(\beta^{\perp}\right) .
$$

Let $\Psi(\beta)=\Phi[\rho(\beta)]$ for $\beta \in \tilde{N}_{1}$. Since $0<h=\operatorname{dim} U_{1}$, where $U_{1}=\operatorname{Ker} \Phi\left(\alpha_{0}\right)$, there exists $\epsilon>0$ such that all nonzero eigenvalues of $\Phi\left(\alpha_{0}\right)=\Psi(0)$ are $>2 \epsilon$. Using the Cauchy integral formula for matrices, integrating for a projection around the circle of radius $\epsilon$ about 0 , we see that in some neighbourhood of 0 , the orthogonal projection $\pi_{1 \beta}$ of $U_{1 \beta}$ is an analytic function of $\beta$, where

$$
U_{1 \beta}=\sum \bigoplus\left\{U_{s}: U_{s} \text { is eigenspace of eigenvalue } s \text { of } \Psi(\beta) \text {, and }|s|<\epsilon\right\} \text {. }
$$

Defining $U_{2 \beta}=U_{1 \beta}^{1}, \pi_{2 \beta}=I-\pi_{1 \beta}$, we can use the Gram-Schmidt process on $\left\{\pi_{j \beta} \beta_{j}^{k}\right\}$, where $\left\{\beta_{j}^{k}\right\}$ is a fixed orthonormal basis of $U_{j}=U_{j 0}$, to define a unitary map $R_{j \beta}: U_{j} \rightarrow U_{j \beta}$ which is an analytic function of $\beta$. Setting $R_{\beta}=R_{1 \beta} \oplus R_{2 \beta}$, we see that $R_{\beta}: \mathrm{C}^{n} \rightarrow \mathrm{C}^{n}$ is unitary and analytic in $\beta$, and $R_{0}=I$.

Let $\left(\begin{array}{ll}0 & 0 \\ 0 & C\end{array}\right)$ be the $\left(U_{1}, U_{2}\right)$-block matrix expression for $\Psi(0)$, and let

$$
\left(\begin{array}{ll}
A_{1} & B_{1} \\
B_{1}^{*} & C_{1}
\end{array}\right)=\left(\frac{\partial}{\partial \beta_{1}} \Psi\right)(0) .
$$

Since in addition to (68) we may assume $\left(\beta_{1}, \beta^{1}\right) \in \tilde{N}_{2} \cap \rho^{-1}\left(\bar{\Gamma}^{*}\right) \Rightarrow \beta_{1} \geqslant 0$, it follows that $\Psi[(t, 0,0, \ldots, 0)]>0$ for $0<t<t_{0}>0$ and hence $A_{1}>0$; also $C>0$ by definition of $U_{1}$. Expanding, we see that

$$
\left.\frac{\partial}{\partial \beta_{1}} R_{\beta}^{*} \Psi(\beta) R_{\beta}\right|_{\beta=0}=\left(\begin{array}{cc}
A_{1} & 0 \\
0 & \tilde{C}
\end{array}\right)
$$

for some matrix $\tilde{C}$, since off-diagonal blocks of $R_{\beta}^{*} \Psi(\beta) R_{\beta}$ are identically zero. By continuity, the upper left-hand block of $\left(\partial / \partial \beta_{1}\right) R_{\beta}^{*} \Psi(\beta) R_{\beta}$ is $>0$ for $\beta$ in some neighbourhood of 0 .

Thus, assuming $\widetilde{N}_{1}$ and $\widetilde{N}_{2}$ small enough, we have by the assumption of (f) an analytic function $\theta: \widetilde{N}_{1} \rightarrow \mathbf{R}$ such that

$$
\begin{gathered}
\partial \theta(\beta) / \partial \beta_{1}>0 \text { and } \theta(\beta)^{h}=\operatorname{det} \Psi(\beta) \text { for } \beta \in \tilde{N}_{1}, \\
\left\{\beta \in \tilde{N}_{1}: \theta(\beta)=0\right\}=\left\{\beta \in \tilde{N}_{1}: \beta_{1}=\beta_{1}\left(\beta^{\perp}\right), \beta^{\perp} \in N_{2}\right\} .
\end{gathered}
$$

It follows that $\beta_{1}\left(\beta^{\perp}\right)$ is analytic. Since $k^{*}(p)=1$, we have $\left\{\beta^{\perp} \in \widetilde{N}_{2}\right.$ : $\left.\beta_{1}\left(\beta^{\perp}\right)=0\right\}=\{0\}$; since $p$ is a "nice" point of $\bar{\Gamma}$, the Hessian of $\beta_{1}\left(\beta^{\perp}\right)$ at $\beta^{\perp}=$ 0 must be positive definite. It follows as in [1] that $r(p)=m-2, k(p)=1$, 
and $\partial \Gamma$ is analytic near $p$. Thus 2.3(a) and 2.3(b) are satisfied $[1,2.17]$. Taking the derivative as in 2.2, one finds that $\operatorname{dim} \operatorname{Ker} \Phi_{0}(\alpha)=h$ for $\alpha \in \partial \Gamma_{0}^{*}, \tau_{3}(\alpha)$ $\neq 0$; from this it follows as in (23) that $2.3(\mathrm{c})$ holds near zero by continuity (since $C(\alpha)>0$ ), and hence everywhere by analyticity.

4.5. Conjecture. We conjecture that the conclusion of Theorem 3.5 holds at $p \in \bar{\Gamma}$, where $r(p)=0$, and $\bar{\Gamma}$ is formed by direct summations, linear transformations, and special intersections $[1,1.5]$ from cones $\bar{\Gamma}_{j}$ of the following types:

(a) $\Gamma_{j}+i\left[\Gamma_{j}\right]$ is the infinite realization of a Hermitian symmetric space; or

(b) $\Gamma_{j}, \Phi_{j}$ satisfy the hypotheses of $4.4(\mathrm{f})$ at every $p_{j} \in \partial \Gamma_{j}-\{0\}$. Here we assume $\Phi$ is constructed from the $\Phi_{j}$ by direct summations and linear transformations as in 4.4(b), (c), and left fixed under special intersections.

REMARKS. It is clear by the usual arguments (see [1]) that if we can prove the kernels in 4.5(a), (b) are "radially almost-everywhere fast-decreasing" then the claim follows. Case (a), which is independent of $\Phi_{j}$, should follow as in $\S$ III above by using the group of automorphisms of $\Gamma_{j}$. In case (b), which we can extend to include $h=0$ (i.e. 4.4(d)), the methods of the proof of [1, 3.3], may apply, though the case $h>1$ presents difficulties. In general, the author makes the following conjecture, whose proof would seem to require more advanced methods than those used here:

4.6. Conjecture. The conclusion of Theorem 3.5 holds at $p \in \bar{\Gamma}$ if and only if $r(p)=0$.

\section{BIBLIOGRAPHY}

1. L. J. Dickson, Limit properties of Poisson kernels of tube domains, Trans. Amer. Math. Soc. 182 (1973), 383-401. MR 48 \#9273.

2. A. Koranyi, The Poisson integral for generalized half-planes and bounded symmetric domains, Ann. of Math. (2) 82 (1965), 332-350. MR 34 \#371.

3. A. Korányi and J. A. Wolf, Realization of hermitian symmetric spaces as generalized half-planes, Ann. of Math. (2) 81 (1965), 265-288. MR 30 \#4980.

4. I. I. Pjateckii-Sapiro, Geometry of classical domains and theory of automorphic functions, Fizmatgiz, Moscow, 1961; English transl., Automorphic functions and the geometry of classical domains, Math. and its Applications, vol. 8, Gordon and Breach, New York, 1969. MR 25 \#231; 40 \#5908.

5. E. M. Stein and N. J. Weiss, On the convergence of Poisson integrals, Trans. Amer. Math. Soc. 140 (1969), 35-54. MR $39 \# 3024$.

Further references are found in the bibliographies of [1] and [2].

DEPARTMENT OF MATHEMATICS, UNIVERSITY OF NEW SOUTH WALES, KENSINGTON, NSW 2033, AUSTRALIA

Current address: 12727 3rd N.W., Seattle, Washington 98177 